\title{
Multiple invasions of Gypsy and Micropia retroelements in genus Zaprionus and melanogaster subgroup of the genus Drosophila Nathalia de Setta ${ }^{1}$, Marie-Anne Van Sluys ${ }^{2}$, Pierre Capy ${ }^{3}$ and Claudia MA Carareto*1
}

Address: ${ }^{1}$ UNESP - São Paulo State University, Department of Biology, São José do Rio Preto, SP, Brazil, 2 USP - São Paulo University, Department of Botany, São Paulo, SP, Brazil and 32Laboratoire Evolution, Génomes et Spéciation UPR9034, CNRS, 91198 Gif-sur-Yvette, and Université ParisSud 11, 91405 Orsay, France

Email: Nathalia de Setta - nsetta@hotmail.com; Marie-Anne Van Sluys - mavsluys@usp.br; Pierre Capy - pierre.capy@legs.cnrs-gif.fr; Claudia MA Carareto* - carareto@ibilce.unesp.br

* Corresponding author

Published: 2 December 2009

BMC Evolutionary Biology 2009, 9:279 doi:10.1 |86//47|-2|48-9-279
Received: II June 2009

Accepted: 2 December 2009

This article is available from: http://www.biomedcentral.com/I47I-2/48/9/279

(c) 2009 de Setta et al; licensee BioMed Central Ltd.

This is an Open Access article distributed under the terms of the Creative Commons Attribution License (http://creativecommons.org/licenses/by/2.0), which permits unrestricted use, distribution, and reproduction in any medium, provided the original work is properly cited.

\begin{abstract}
Background: The Zaprionus genus shares evolutionary features with the melanogaster subgroup, such as space and time of origin. Although little information about the transposable element content in the Zaprionus genus had been accumulated, some of their elements appear to be more closely related with those of the melanogaster subgroup, indicating that these two groups of species were involved in horizontal transfer events during their evolution. Among these elements, the Gypsy and the Micropia retroelements were chosen for screening in seven species of the two Zaprionus subgenera, Anaprionus and Zaprionus.

Results: Screening allowed the identification of diverse Gypsy and Micropia retroelements only in species of the Zaprionus subgenus, showing that they are transcriptionally active in the sampled species. The sequences of each retroelement were closely related to those of the melanogaster species subgroup, and the most parsimonious hypothesis would be that 15 horizontal transfer events shaped their evolution. The Gypsy retroelement of the melanogaster subgroup probably invaded the Zaprionus genomes about II MYA. In contrast, the Micropia retroelement may have been introduced into the Zaprionus subgenus and the melanogaster subgroup from an unknown donor more recently ( 3 MYA).

Conclusion: Gypsy and Micropia of Zaprionus and melanogaster species share similar evolutionary patterns. The sharing of evolutionary, ecological and ethological features probably allowed these species to pass through a permissive period of transposable element invasion, explaining the proposed waves of horizontal transfers.
\end{abstract}

\section{Background}

The Zaprionus genus (Diptera, Drosophilidae) is composed by two subgenera (Anaprionus s.s. and Zaprionus s.s.) which seem to be originated in Asia about 14 MYA
[1]. The Anaprionus subgenus has first diversified in the Oriental biogeographic region, being relatively less studied. However, the Zaprionus subgenus ancestor(s) originated during the Middle to Early Miocene in Oriental 
regions before diversifying in Tropical Africa, sharing space and time with the best studied drosophilid species, the melanogaster subgroup [1,2]. Due to its evolutionary history, ecological and morphological diversity, the Zaprionus genus seems to be a good model for comparative studies with the melanogaster subgroup. Although the phylogenetic relationships within the Zaprionus genus had been recently proposed [1], its taxonomic positioning in the Drosophilidae family remains a matter for discussion. Currently, most reports agree that Zaprionus belongs to the Drosophila genus, and that it would be more closely related to Drosophila than to Sophophora subgenera [3-10], to which the species of the melanogaster subgroup belong (Figure 1). Hence, Zaprionus and melanogaster species would share a last common ancestor at least as old as the divergence of the Sophophora and Drosophila subgenera, i.e., between 35 and 60 MYA $[5,11]$.

While the melanogaster species subgroup - mainly the two sibling species D. melanogaster and D. simulans - presents the most well known dipteran mobilomes, knowledge about the transposable elements (TEs) content of the Zaprionus species is scant. Only seven TE families have been identified in all Zaprionus genus. The elements 412 of $Z$. tuberculatus, 731 of $Z$. ornatus, Bari- 1 of $Z$. tuberculatus and, Mariner of 12 Zaprionus species could only be detected by Southern blot hybridization using probes derived from $D$. melanogaster [12-17]. Furthermore, partial sequences were produced for Gypsy of Z. indianus, Copia of Z. tuberculatus, Mariner of $Z$. tuberculatus and $Z$. verruca, and Rover of $Z$. indianus [17-20]. Interestingly, evolutionary analyses demonstrated that four of these seven TEs of Zaprionus are highly related to those the melanogaster subgroup, sharing their common ancestor more recently than the species ones. Gypsy, Copia, Mariner and Rover were therefore proposed to have been horizontally transferred between species of the Zaprionus subgenus and the melanogaster subgroup of the genus Drosophila [15-18,20,21]. Horizontal transfer (HT) of TEs has been inferred when three criteria are met: (a) high sequence conservation between TE sequences of distantly related species; (b) incongruence between TE and host phylogenies and, (c) discontinuous distribution of a TE across a species group. Additionally, requirements like geographic, temporal and ecological overlapping and, exclusion of alternative hypothesis as ancestral polymorphism promoting differential subfamily fixation and high selective constraints over the sequences need to be considered [22].

In a previous study, we have performed a search of $D$. melanogaster TEs in the American continent invasive species $Z$. indianus by Dot blot methodology, aiming to broaden our understanding of TE occurrence in species of the Zaprionus genus [23]. All the 10 TEs analyzed were present in the $Z$. indianus genome, but only five had strong hybridization signals with the probes that were used: Roo/B104, Doc, MDG-1, Micropia and Gypsy. Among them, we selected Gypsy and Micropia retroelements to expand our evolutionary analyses in the Zaprionus genus. This choice was due to the evidence of the close relationship of these

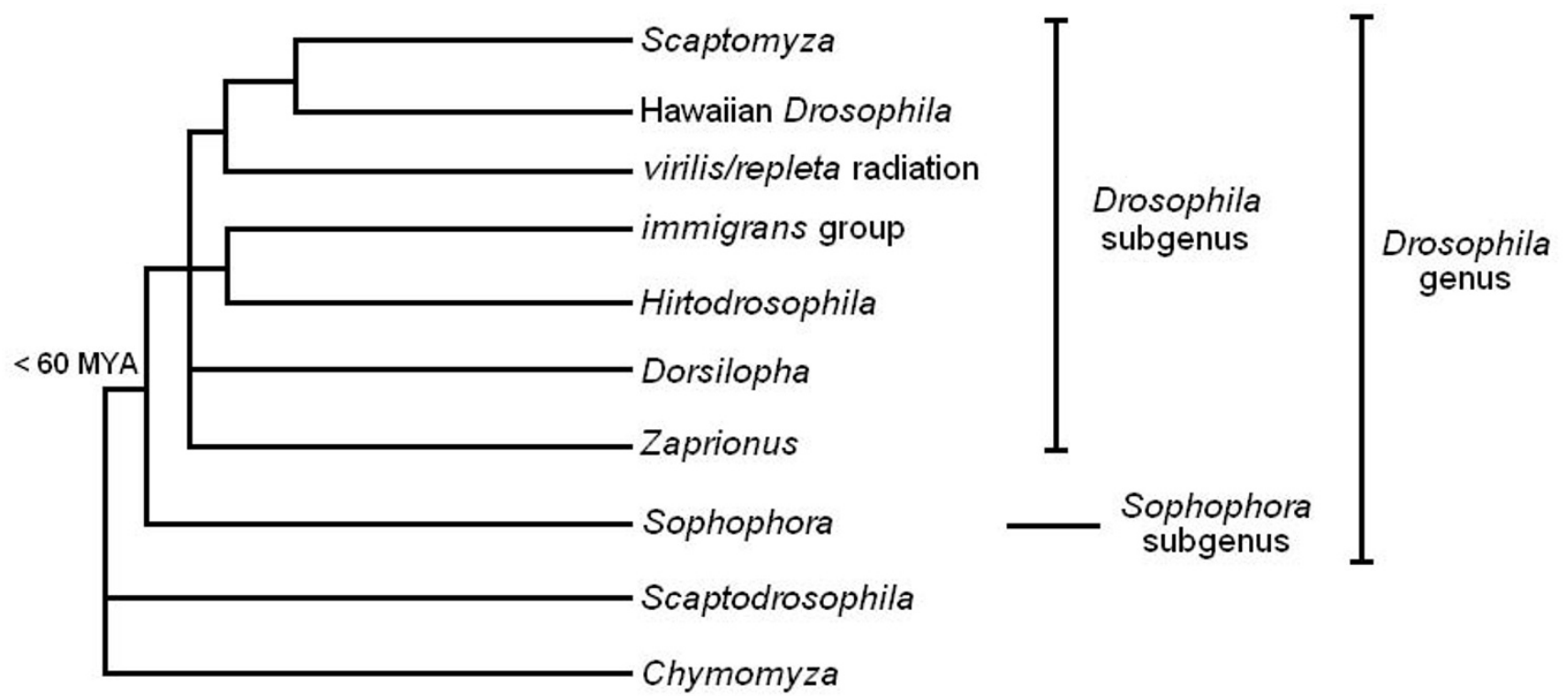

Figure I

Phylogenetic tree of the Drosophilidae family. Phylogenetic relationships between the main Drosophilidae species groups, evidencing the taxonomic positioning of the Zaprionus genus inside the Drosophila subgenus and the divergence time between Drosophila and Sophophora subgenus [redrawn from reference [7]]. 
Z. indianus elements with others already described in the literature, inferred by the strong hybridization signals using heterologous probes, the easy amplification with the primers available in the literature and the proposition of a Gypsy HT of between $Z$. indianus and D. simulans [18].

Gypsy and Micropia are classified as Ty3/Gypsy elements because of their gene arrangement in the pol coding region - Reverse transcriptase/RNAseH/Integrase [24]. Ty3/Gypsy retroelements, which are similar to vertebrate retroviruses in both sequence and genomic structure, are broadly distributed among eukaryotes [25]. They differ from vertebrate retroviruses mainly because they do not have an env gene necessary to complete the infectious cycle [24]. Gypsy is an exception to the typical Ty3/Gypsy structure because an active env is present, potentially capable of maintaining infectivity [26]. Hence, Gypsy was classified as an Errantivirus by the International Committee on Taxonomy of Viruses (ICTV) [27].

The Micropia retroelement had already been studied in $D$. melanogaster, and several species of the repleta and the cardini groups of the subgenus Drosophila [28-30]. Evolutionary analyses showed the existence of at least two Micropia families that have $30 \%$ divergence in DNA sequence [29]. The first family, present in the repleta group, comprises two subfamilies than were differentiated by nucleotide divergence and antisense RNA expression. The repleta species group seems to have transmitted one of its elements to the D. cardini group through HT [30]. The second family is represented by the D. melanogaster element that also transcribes antisense RNAs responsible for control of its expression [31]. The data suggest that the Micropia retroelement is an ancient component of Drosophila genomes [29].

In contrast to Micropia, the Gypsy retroelement has been analyzed in the 12 Drosophila genomes [32], and individual sequences from several Drosophilidae species are available, such as the virilis and repleta groups of subgenus Drosophila; the willistoni and melanogaster groups of the subgenus Sophophora; and the Scaptodrosophila latifasciaeformis and the $Z$. indianus species [18,32-34]. Two Gypsy families have been identified, one exclusive to $D$. willistoni and the other widely distributed among Drosophilidae species [18]. The latter is divided into 10 subfamilies, seven of which have been implicated in HT events, demonstrating that inheritance of the Gypsy retroelement is complex [18,32]. HT events involving Gypsy could have been facilitated by the presence of env, assumed to confer autonomous infectivity on the retroelement, thereby allowing it to cross species barriers $[18,32,35]$. Specifically in the melanogaster species subgroup, two Gypsy subfamilies have been identified, and seven HTs have been proposed among melanogaster species subgroups, or between one species of that subgroup and S. latifasciaeformis and $Z$. indianus $[18,32]$. These inter-genera HTs are assumed to have occurred because synonymous sites of Gypsy env gene are three to five times more highly conserved than those of host genes in species that diverged longer than 40 MYA [18].

We have now surveyed the Gypsy and Micropia retroelements in seven species of the Zaprionus genus, comprising one species of the Anaprionus subgenus and six of the Zaprionus subgenus. Our objective was to investigate the distribution and evolutionary history of these two elements in this scarcely studied genus by comparing their sequences with those of the 12 Drosophila genomes and other sequences available in the nucleotide database. The results show that the Zaprionus Gypsy and Micropia retroelements are present and transcriptionally active only in the subgenus Zaprionus, to which they were introduced by more than one horizontal transfer event from different donor species.

\section{Results \\ Distribution of Gypsy and Micropia in the genus Zaprionus}

In an attempt to search for the presence of Gypsy and Micropia retroelements in seven species of the Zaprionus genus (Table 1), PCR reactions using primers that amplify Gypsy env and Micropia RNAseH sequences were carried out (see Materials and Methods). This revealed the presence of these elements in the six species of the Zaprionus subgenus, but not in Z. multistriatus (Anaprionus subgenus). Three clones of each Gypsy and Micropia PCR fragments were sequenced and used in the evolutionary analyses. Additionally, RT-PCR reactions and Southern blot hybridization showed that all Zaprionus subgenus species analyzed harbor a few transcriptionally active insertions of Gypsy and Micropia elements, varying from one ( $Z$. gabonicus) to seven ( $Z$. indianus and $Z$. davidi) insertions for Gypsy, and from two (Z. tuberculatus and $Z$. africanus) to seven ( $Z$. indianus) for Micropia [Additional files 1 and 2].

\section{Evolutionary analyses of the Gypsy retroelement}

To infer the evolutionary history of Gypsy retroelement in the Zaprionus subgenus, we carried out genomic searches for Zaprionus homologous sequences in the 12 Drosophila genomes and the GenBank database. The in silico search retrieved genomic sequences only from the melanogaster subgroup, indicating that the other seven Drosophila genomes (D. ananassae, D. pseudoobscura, D. persimilis, $D$. willistoni, D. mojavensis, D. virilis and D. grimshawi) do not harbor Gypsy sequences closely related to those of the subgenus Zaprionus. Eight sequences were identified in $D$. melanogaster, nine in D. simulans, 19 in D. sechellia, six in D. yakuba, and 13 in D. erecta [Additional file 3]. A BLAST- 
Table I: Zaprionus species used in this study, taxonomic classification, divergence time, geographic origin of the strains and GenBank sequence accession numbers.

\begin{tabular}{|c|c|c|c|c|}
\hline \multirow[b]{2}{*}{ Species } & \multirow[b]{2}{*}{$\begin{array}{l}\text { Divergence time }[\mathrm{I}] \\
\text { (MYA) }\end{array}$} & \multirow[b]{2}{*}{ Strain origin } & \multicolumn{2}{|c|}{ GenBank accessions } \\
\hline & & & Gypsy & Micropia \\
\hline Genus Zaprionus & |3.8 (|0.9-|4.9) & & & \\
\hline Subgenus Anaprionus & $10.6(7.7-12.1)$ & & & \\
\hline Z. multistriatus & nd & Bangalore (India) & Absent & Absent \\
\hline Subgenus Zaprionus & $7.4(6.7-9.0)$ & & & \\
\hline Group inermis & 7.0 & & & \\
\hline tuberculatus complex & $3.0(2.9-18.0)$ & & & \\
\hline Z. tuberculatus & $1.1(0.6-1.6)$ & Ithala (South Africa) & F]710406 toF]710408 & F]7I0423 toF]7I0425 \\
\hline Group armatus & nd & & & \\
\hline lachaisei complex & $0.9(0.4-1.1)$ & & & \\
\hline Z. camerounensis & nd & Amani (Tanzania) & F]7I0409 toF]7I04II & F]710426 toFj710428 \\
\hline davidi complex & $2.2(1.4-2.4)$ & & & \\
\hline Z. davidi & $2.2(1.4-2.4)$ & São Tomé (São Tomé e Príncipe) & F]7104I2 toFj7I04I4 & F]710429 toFl710431 \\
\hline indianus complex & $3.1(2.6-4.1)$ & & & \\
\hline Z. gabonicus & nd & Makokou (Gabon) & F]7104I5 toFj7104I7 & F]710432 toFj710434 \\
\hline Z. africanus & nd & Kibale (Uganda) & F]710418 toF]710420 & FJ710435 toFl710437 \\
\hline Z. indianus & nd & Brasília (Brazil) & Fj7I042I toFj7I0422 & FJ710438 toFj710440 \\
\hline
\end{tabular}

nd: not determined.

based analysis of the structure sequence indicated that only D. melanogaster genome presents putatively fulllength insertions [Additional file 4].

\section{Inferring phylogenetic relationships and divergence}

The phylogenetic relationships inferred using the Maximum Parsimony (MP), Maximum Likelihood (ML) and Neighbor-joining (NJ) methods of phylogenetic reconstruction produced similar patterns with identical main branches. The analyses were performed with three sequences of each Zaprionus species and 52 sequences of species from the melanogaster subgroup. The S. latifasciaeformis and D. willistoni Gypsy sequences available in the GenBank database were included in these reconstructions because of their respectively closer and more distant evolutionary relationships to those of the melanogaster subgroup [18]. Figure 2 shows the tree reconstructed by ML analysis. The sequences of the genus Zaprionus, the melanogaster species subgroup and S. latifasciaeformis were grouped together in three clades that were not congruent with the species phylogeny. Clade 1 (bootstrap 97\%) contains sequences of D. melanogaster, D. erecta, D. simulans and only one sequence of the genus Zaprionus, $Z$. tuberculatus 1 . Clade 2 (bootstrap $84 \%$ ) includes $Z$. tuberculatus, Z. camerounensis, S. latifasciaeformis, D. melanogaster, D. erecta and all sequences of D. yakuba, except $D$. yakubaA6, which branches outside the 3 clades. The reported clustering of $D$. erecta and $S$. latifasciaeformis has been explained as an HT event [31]. Finally, clade 3 comprises the Gypsy sequences of $Z$. davidi, $Z$. indianus and the two cryptic species, $Z$. gabonicus and $Z$. africanus, as well as sequences of D. melanogaster, D. simulans and all sequences of $D$. sechellia, with no bootstrap support. Additionally, a tree was reconstructed using the Zaprionus sequences, the genomic sequences of melanogaster subgroup, and Gypsy sequences of 17 other Drosophila species (from the guarani, cardini, pallidipennis, tripunctata, repleta, anulimana and flavopilosa groups of the subgenus Drosophila and D. busckii [18]). A similar topology of the Figure 2 was obtained, with all Zaprionus sequences clustering together with melanogaster elements in the same clade distribution, which reinforces the close relationship between Zaprionus and melanogaster Gypsy sequences (data not shown).

Distance analysis shows that the sequences within the three clades have relatively low divergence levels, with mean values of $0.063,0.070$ and 0.042 within clades 1,2 and 3, respectively (Table 2 and Additional file 5). The distances of the Zaprionus vs melanogaster species within each group are similar to the mean values just cited. The mean distances between the clades show that clade 2 is closer to clade 3 than to clade 1 , with mean values of 0.119 (clade 2 vs clade 3 ), 0.239 (clade 1 vs clade 2 ) and 0.241 (clade 1 vs clade 3). The Drosophila Gypsy sequences, which vary up to $20 \%$ in nucleotide composition, were previously classified into 10 subfamilies [18,32]. Among them, the sequences of $Z$. indianus and the melanogaster species subgroup were included in two different subfamilies. The addition of 18 sequences belonging to six Zaprionus species allowed us to confirm this proposition. The species distribution and divergence of the clade 1 


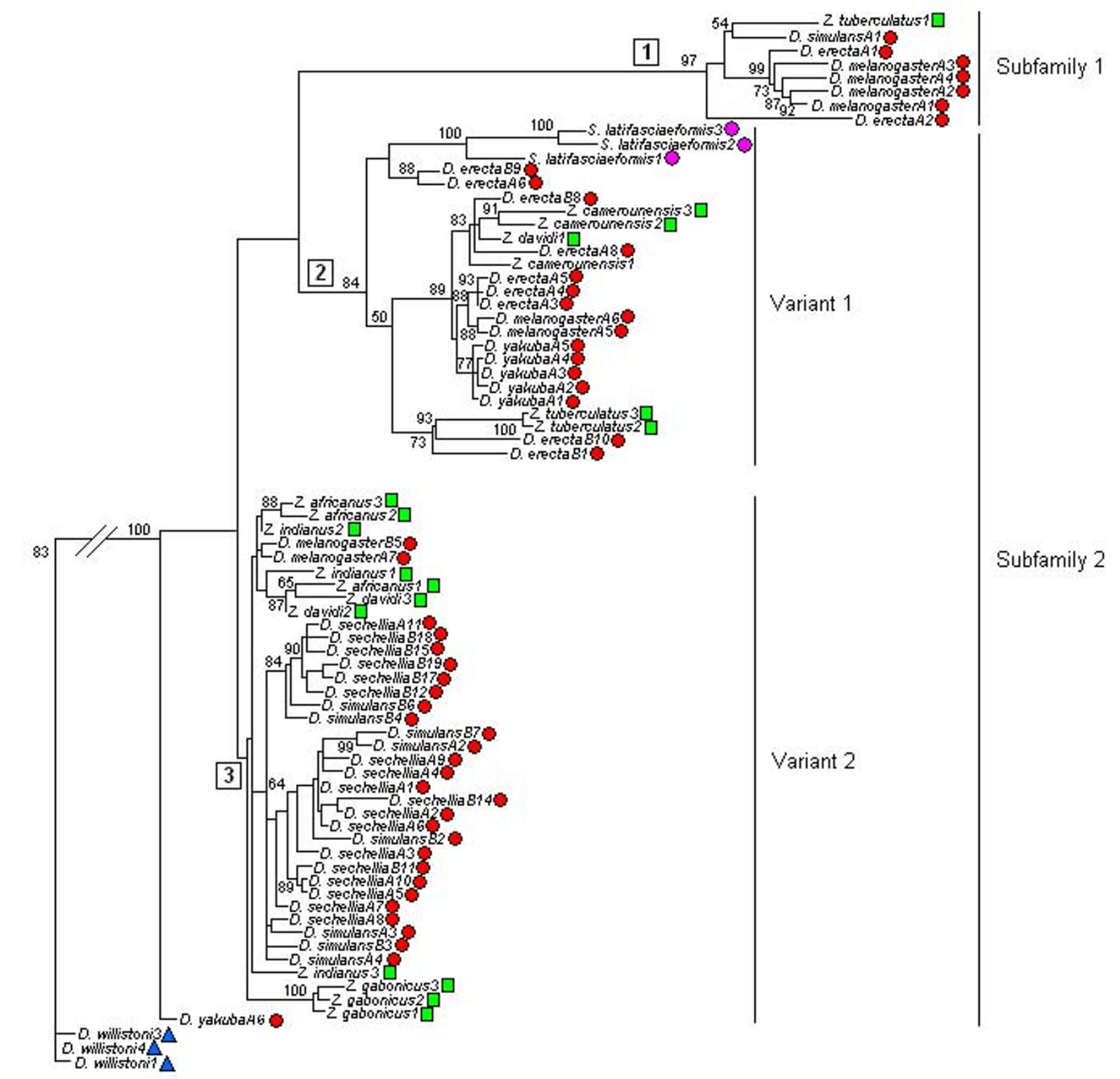

$\underline{0.1}$

Figure 2

Phylogenetic relationships between Gypsy retroelements. Phylogeny of Gypsy sequences of genus Zaprionus (green squares), the melanogaster subgroup (red circles), D. willistoni (blue triangles) and S. latifasciaeformis (pink circles). Numbers in squares indicate the clade number mentioned in the text. The tree was reconstructed using the maximum-likelihood method (HKY85 distance), as implemented in the PhyML program. The branch support was calculated using bootstrap test (I000 replications). Numbers next to species names indicate the clone or genomic sequence identification. 
Table 2: Genetic divergence between the Gypsy and Micropia retroelements

\begin{tabular}{|c|c|c|c|c|}
\hline Retroelement & $\mathbf{N}$ & Mean & Minimum & Maximum \\
\hline \multicolumn{5}{|l|}{ Gypsy } \\
\hline clade I - SI & 28 & 0.0628 & 0.0213 & 0.1112 \\
\hline Zaprionus vs melanogaster SI & 7 & 0.0742 & 0.0529 & 0.1006 \\
\hline clade 2 - S2VI & 300 & 0.0704 & 0.0021 & 0.1687 \\
\hline Zaprionus vs melanogaster VI & 96 & 0.0641 & 0.0211 & 0.1122 \\
\hline clade 3 - S2V2 & 741 & 0.0422 & 0.0042 & 0.0888 \\
\hline Zaprionus vs melanogaster V2 & 308 & 0.0506 & 0.0086 & 0.0888 \\
\hline clade I (SI) vs clade $2(\mathrm{VI})$ & 200 & 0.2388 & 0.2080 & 0.2910 \\
\hline clade I (SI) vs clade 3 (V2) & 312 & 0.2406 & 0.2055 & 0.286 \\
\hline clade 2 (VI) vs clade 3 (V2) & 975 & 0.1189 & 0.0771 & 0.1814 \\
\hline \multicolumn{5}{|l|}{ Micropia } \\
\hline clade I & 66 & 0.0091 & 0 & 0.0216 \\
\hline clade 2 & 496 & 0.0509 & 0 & 0.1473 \\
\hline clade I vs clade 2 & 384 & 0.0739 & 0.0288 & 0.1341 \\
\hline Zaprionus $\times$ melanogaster group & 468 & 0.0727 & 0.0188 & 0.1341 \\
\hline Zaprionus genus & 153 & 0.0328 & 0 & 0.0850 \\
\hline melanogaster group & 325 & 0.0466 & 0 & 0.1473 \\
\hline
\end{tabular}

Note: The MCL pairwise distance values between the Zaprionus subgenus and melanogaster species subgroup were summarized according the Gypsy subfamilies and variants and, species groups for Micropia. SI - Subfamily I; S2 - Subfamily 2; VI - Variant I; V2 - Variant 2. N: number of pairwise comparisons.

from 2 and $3(0.24)$ indicate that it corresponds to one of those subfamilies previously proposed, hereafter called subfamily 1 (S1). On the other hand, a divergence of 0.119 between clades 2 and 3 led us to classify them as variants 1 and 2 (V1 and V2) of another subfamily (S2) that may correspond with the second subfamily already described due to its species composition.

\section{Testing the HT hypothesis}

Three hypotheses could explain the high levels of conservation among Gypsy sequences within each clade (0.040.07 ) and the phylogenetic inconsistencies compared to the species tree: (a) high selective constraints conserving the sequences; (b) ancestral polymorphism promoting differential subfamily fixation in the species; and (c) the occurrence of HT. To test whether (a) can best explain the estimated conservation, the ratio of non-synonymous divergence per synonymous divergence ( $\mathrm{dN}: \mathrm{dS}$ ) within the clades were compared to those of the Gpdh nuclear gene, offering a comparative measurement of the selective constraints. Although active elements might suffer selective pressure to maintain their coding sequences, Gpdh would be expected to be even more restricted because it plays an essential role in glycerophospholipid metabolism in Drosophila. If it is assumed that synonymous substitutions are under almost strictly neutral evolution, $\mathrm{dN}: \mathrm{dS}=1, \mathrm{dN}: \mathrm{dS}<1$ and $\mathrm{dN}: \mathrm{dS}>1$ will represent neutral evolution, purifying selection and positive Darwinian selection, respectively. The analyses indicate that the mean Gypsy dN:dS ratios ranged from 0.153 to 1.002, while the mean Gpdh values did not exceed 0.041 (Table
3, and Additional file 6). These ratios suggest that, although $\mathrm{dN}$ : $\mathrm{dS}<1$, purifying selection over the Gypsy env sequences is relaxed $(\mathrm{dN}: \mathrm{dS}$ mean $=0.395)$ compared to the host gene. Also, a Z-test for the Gypsy env region indicates neutrality for most of the pairwise comparisons $(62 \%)$ within the clades [Additional file 7]. Hence, high selective constraints do not explain the incongruities observed intra-clades. On the other hand, the neutrality hypothesis was refuted for all pairwise comparisons between the clades, indicating that purifying selection could plays a role in the conservation of the env coding sequence among the Gypsy subfamilies. In that case, natural selection could act at the genomic level, conserving the TE genes, since elements that transpose most efficiently, or at the highest rate are most likely to survive and propagate [36].

In the absence of intra-clade selective constraints, the comparisons between the dS values of the TE and a host gene permit evaluation of the hypothesis of ancestral polymorphism within subfamilies. If the Gypsy retroelement is evolving vertically and the incongruence in the phylogeny are due to differential subfamily distribution in the analyzed species, the dS pairwise comparisons between the TE and the host gene would be expected to be equivalent. But, if the dS of TE is lower than the dS of host gene under similar or higher levels of selective constraints and in the absence of strong codon usage bias, HT events can be inferred. Using this approach, the dS of Gypsy and Gpdh gene, as well as the Codon Bias Index (CBI) were compared for one clone representing each species of Zap- 
Table 3: Comparative analyses of synonymous divergence (dS), non-synonymous divergence (dN), dN:dS ratios and codon bias index (CBI) of the Gypsy and Micropia sequences and Gpdh.

\begin{tabular}{|c|c|c|c|c|}
\hline Sequences & CBI mean (min-max) & dN mean (SE) & dS mean (SE) & dN:dS mean (SE) \\
\hline Gypsy & $\begin{array}{c}0.4 I 4 \\
(0.344-0.523)\end{array}$ & & & \\
\hline \multicolumn{5}{|l|}{ Subfamily I } \\
\hline Zaprionus $\times$ melanogaster spp. & & $\begin{array}{c}0.045 \\
\left(9.70 \times 10^{-8}\right)\end{array}$ & $\begin{array}{c}0.128 \\
\left(5.95 \times 10^{-4}\right)\end{array}$ & $\begin{array}{c}0.374 \\
(0.004)\end{array}$ \\
\hline melanogaster spp. & & $\begin{array}{c}0.035 \\
\left(3.88 \times 10^{-5}\right)\end{array}$ & $\begin{array}{c}0.057 \\
\left(6.49 \times 10^{-4}\right)\end{array}$ & $\begin{array}{l}1.002 \\
(0.465)\end{array}$ \\
\hline \multicolumn{5}{|l|}{ Subfamily 2 - VI } \\
\hline Zaprionus $\times$ melanogaster spp. & & $\begin{array}{c}0.028 \\
\left(3.55 \times 10^{-5}\right)\end{array}$ & $\begin{array}{l}0.176 \\
(0.002)\end{array}$ & $\begin{array}{c}0.189 \\
(0.005)\end{array}$ \\
\hline Zaprionus spp. & & $\begin{array}{c}0.032 \\
\left(4.42 \times 10^{-5}\right)\end{array}$ & $\begin{array}{c}0.177 \\
(0.004)\end{array}$ & $\begin{array}{l}0.310 \\
(0.019)\end{array}$ \\
\hline melanogaster spp. & & $\begin{array}{c}0.019 \\
\left(4.85 \times 10^{-5}\right)\end{array}$ & $\begin{array}{l}0.119 \\
(0.002)\end{array}$ & $\begin{array}{l}0.153 \\
(0.002)\end{array}$ \\
\hline \multicolumn{5}{|l|}{ Subfamily $2-$ V2 } \\
\hline Zaprionus $\times$ melanogaster spp. & & $\begin{array}{c}0.028 \\
\left(2.65 \times 10^{-5}\right)\end{array}$ & $\begin{array}{c}0.106 \\
\left(2.62 \times 10^{-4}\right)\end{array}$ & $\begin{array}{c}0.291 \\
(0.004)\end{array}$ \\
\hline Zaprionus spp. & & $\begin{array}{c}0.027 \\
\left(2.50 \times 10^{-5}\right)\end{array}$ & $\begin{array}{c}0.088 \\
\left(2.55 \times 10^{-4}\right)\end{array}$ & $\begin{array}{l}0.365 \\
(0.011)\end{array}$ \\
\hline melanogaster spp. & & $\begin{array}{c}0.018 \\
\left(1.10 \times 10^{-6}\right)\end{array}$ & $\begin{array}{c}0.070 \\
\left(1.90 \times 10^{-4}\right)\end{array}$ & $\begin{array}{l}0.477 \\
(0.013)\end{array}$ \\
\hline Micropia & $0.467(0.4 \mid 0-0.527)$ & & & \\
\hline Zaprionus $\times$ melanogaster spp. & & $\begin{array}{c}0.045 \\
\left(1.44 \times 10^{-5}\right)\end{array}$ & $\begin{array}{c}0.124 \\
\left(1.94 \times 10^{-4}\right)\end{array}$ & $\begin{array}{c}0.524 \\
(0.015)\end{array}$ \\
\hline Zaprionus spp. & & $\begin{array}{c}0.026 \\
\left(3.80 \times 10^{-5}\right)\end{array}$ & $\begin{array}{c}0.040 \\
\left(2.45 \times 10^{-4}\right)\end{array}$ & $\begin{array}{c}0.579 \\
(0.019)\end{array}$ \\
\hline melanogaster spp. & & $\begin{array}{c}0.028 \\
\left(6.45 \times 10^{-5}\right)\end{array}$ & $\begin{array}{c}0.068 \\
\left(4.87 \times 10^{-4}\right)\end{array}$ & $\begin{array}{c}0.388 \\
(0.005)\end{array}$ \\
\hline Gpdh & $0.602(0.492-0.700)$ & & & \\
\hline Zaprionus $\times$ melanogaster spp & & $\begin{array}{c}0.009 \\
\left(3.07 \times 10^{-6}\right)\end{array}$ & $\begin{array}{l}1.012 \\
(0.004)\end{array}$ & $\begin{array}{c}0.009 \\
\left(4.90 \times 10^{-6}\right)\end{array}$ \\
\hline Zaprionus spp. & & $\begin{array}{c}0.005 \\
\left(2.16 \times 10^{-5}\right)\end{array}$ & $\begin{array}{l}0.150 \\
(0.002)\end{array}$ & $\begin{array}{c}0.041 \\
\left(5.43 \times 10^{-4}\right)\end{array}$ \\
\hline melanogaster spp. & & 0 & $\begin{array}{l}0.193 \\
(0.003)\end{array}$ & 0 \\
\hline
\end{tabular}

Note: Pairwise dS and dN distances in Additional files 3 and 6 .

rionus and the best Blastn subject of the genomic sequences in each clade. Codon usage bias is a factor that may be responsible for low dS values for TE sequences, since there is a negative correlation between codon usage bias and dS values [20]. The Codon Bias Index (CBI) indicates that the Gypsy and Gpdh sequences did not suffer high codon bias, although the mean value of 0.602 for Gpdh suggests that the small difference in magnitude of dS between Gpdh and Gypsy ought to be taken cautiously in inferring HT, since the variation in the Gpdh dS distance could reflect codon usage. Hence, comparisons between Gypsy and Gpdh dS were used to infer HT only if the dS values of Gypsy were at least twice as low as those of Gpdh. The Gypsy dS values between Zaprionus and melanogaster species ranged from 3 (Z. tuberculatus 2 vs D. melanogaster5 and D. erecta3) to 20 times (D. melanogaster7 vs Z. davidi2) lower than those of Gpdh (Figure 3). On the other hand, comparisons of dS between the Zaprionus species within each clade gave Gypsy dS values approximately equal or higher than Gpdh dS, excepting for Z. davidi2 vs Z. indianus1 and Z. africanus1 which presented dS of Gypsy slightly lower than the host gene ones. These results may indicate that the Gypsy retroelement evolved mainly by vertical transmission within the Zaprionus subgenus, but there may have been HT events between the Zaprionus and the melanogaster species what could reject the hypothesis of ancestral polymorphism within the clades.

\section{Phylogenetic relationships of the Micropia retroelement} In order to evaluate the evolutionary history of Micropia, the same approaches of phylogeny and divergence estimations used for Gypsy were applied. Sequences homologous to the Zaprionus species were also identified only in species of the melanogaster subgroup, except for $D$. erecta, the unique species of the melanogaster subgroup for which no significant matches were obtained. D. sechellia had the most hits (22), followed by D. simulans (six), D. yakuba (four) and D. melanogaster (three) [Additional file 3]. Structure analysis indicates that the D. melanogaster and $D$. 

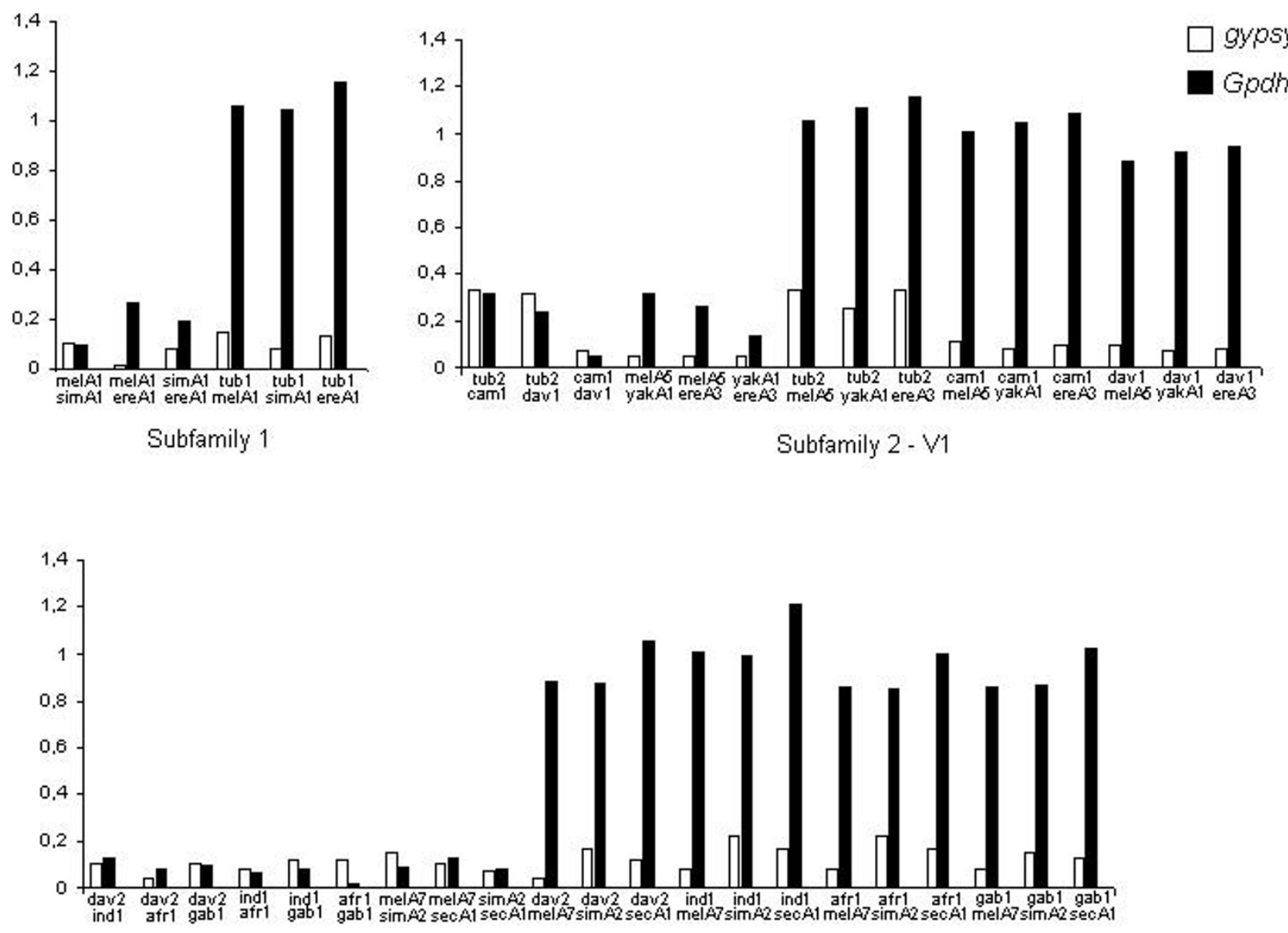

Subfamily $2-\sqrt{ } 2$

\section{Figure 3}

Comparative analysis of dS values of Gypsy and Gpdh. Comparative analyses of the dS values between Gypsy and Gpdh sequences of Zaprionus and melanogaster species within the SI subfamily, S2VI and S2V2. tub: $Z$. tuberculatus, cam: Z. camerounensis, dav: Z. davidi, gab: Z. gabonicus, afr: Z. africanus, ind: Z. indianus, mel: D. melanogaster, sim: $D$. simulans, sec: D. sechellia, yak: D. yakuba, ere: D. erecta.

simulans genomes harbor putatively full-length insertions [Additional file 8].

\section{Inferring phylogenetic relationships and divergence}

The MP, NJ and ML reconstructions of the Micropia sequences from the Zaprionus subgenus, the genomic sequences from the melanogaster subgroup and the GenBank sequences of the repleta group had similar topologies. As for Gypsy, only ML reconstruction is given (Figure 4). The tree exhibits several incongruences according to species phylogeny, such as grouping species in the Zaprionus genus together with those of the melanogaster subgroup. The sequences of the Micropia family of the repleta group were clustered outside a well-supported branch that harbors all sequences of the Zaprionus subgenus and the melanogaster species subgroup, constituted by two clades. Clade 1, with low support (48\%), grouped the $D$. sechelliaA20 sequence with three internal clades, corresponding to the $Z$. camerounensis sequences (bootstrap $98 \%$ ), D. yakuba (no support) and the sequences of $Z$. davidi, Z. indianus, Z. africanus and Z. gabonicus (bootstrap $75 \%$ ). This last clade did not give a clear internal speciesspecific clustering. Clade 2 grouped sequences of the melanogaster subgroup (from D. melanogaster, D. simulans and $D$. sechellia) together with $Z$. tuberculatus in $76 \%$ of the replications. Despite the clustering of all melanogaster sequences in clade 2, the support was not high (52\%). The mean divergence within clade 1 was much lower $(0.009)$ than in clade $2(0.051)$ or between them (0.074) [Additional file 9]. Analyzing the species groups separately, the 


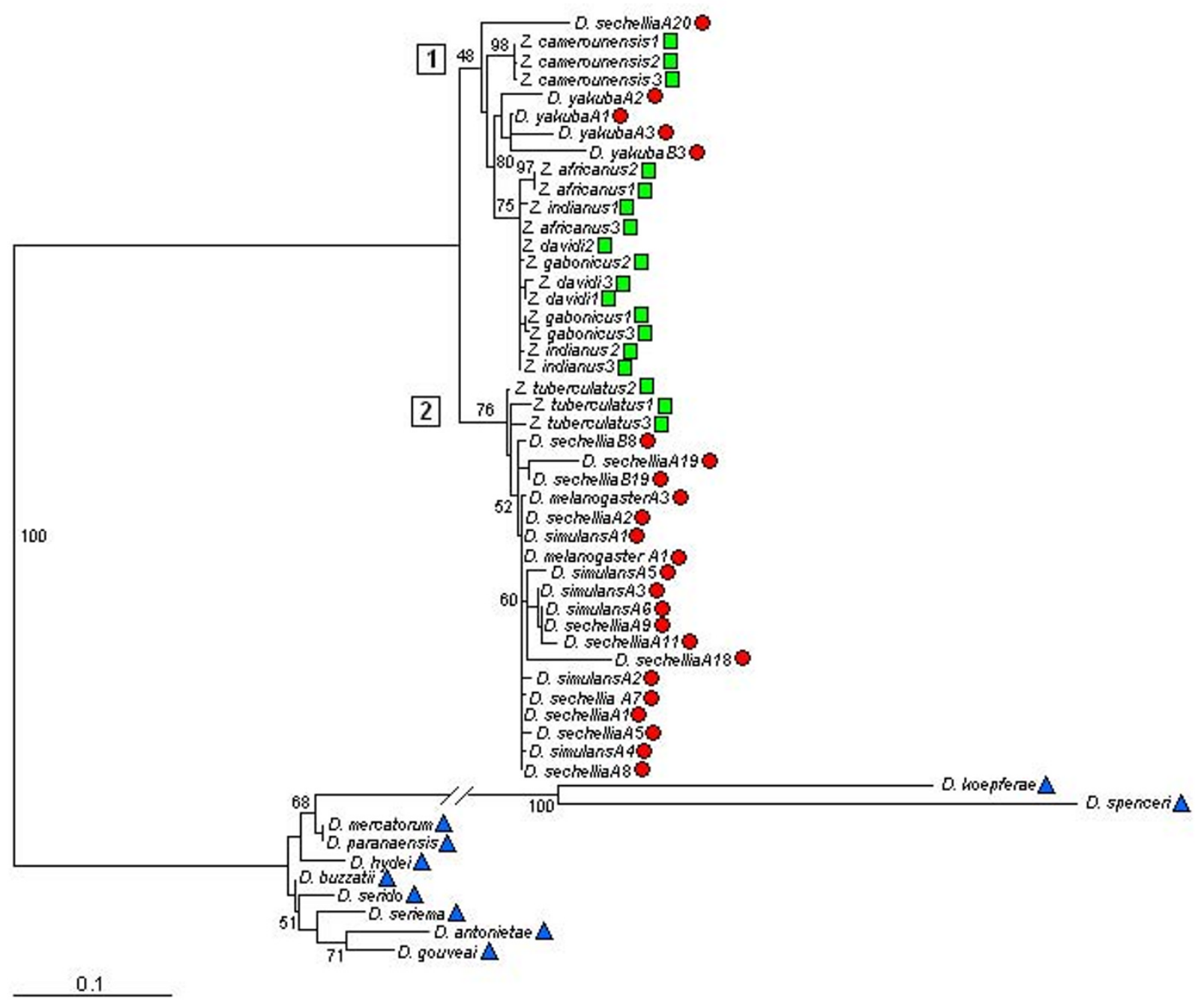

\section{Figure 4}

Phylogenetic relationships between Micropia retroelements. Phylogeny of Micropia sequences of genus Zaprionus (green squares) and the melanogaster (red circles) and repleta (blue triangles) groups. The trees were reconstructed using the maximum-likelihood method (HKY85 distance), as implemented in the PhyML program. Numbers in squares indicate the clade number mentioned in the text. The branch support was calculated using bootstrap test ( 1000 replications). Numbers next to species names indicate the clone or genomic sequence identification.

mean divergence of the Zaprionus sequences was 0.033 ; it was 0.047 within the melanogaster species subgroup, and 0.073 between the Zaprionus and the melanogaster sequences (Table 2). The phylogenetic tree and distance estimates $<20 \%$ indicate that Micropia in the Zaprionus and melanogaster species belong to the same subfamily.

\section{Testing the HT hypothesis}

Evaluation of the selective constraints showed that the mean dN:dS values of the Micropia sequences were 14-58 times higher than those of Gpdh (Table 3 and [Additional file 10]). Between the Zaprionus and melanogaster sequences, for example, the values were 0.524 and 0.009 for Micropia and Gpdh, respectively. In addition, the Z-test failed to reject the neutrality hypothesis in $~ 80 \%$ of the pairwise comparisons [Additional file 11]. These results refute the hypothesis that the high similarity between the Micropia sequences of Zaprionus and melanogaster is due to high selective constraints conserving their nucleotide sequences.

To evaluate the ancestral polymorphism hypothesis for Micropia, the dS values of Micropia RNAseH and Gpdh sequences were compared between the sequences of Zaprionus and melanogaster species in each clade (Figure 5). All the dS distances for the Micropia were lower than those for 


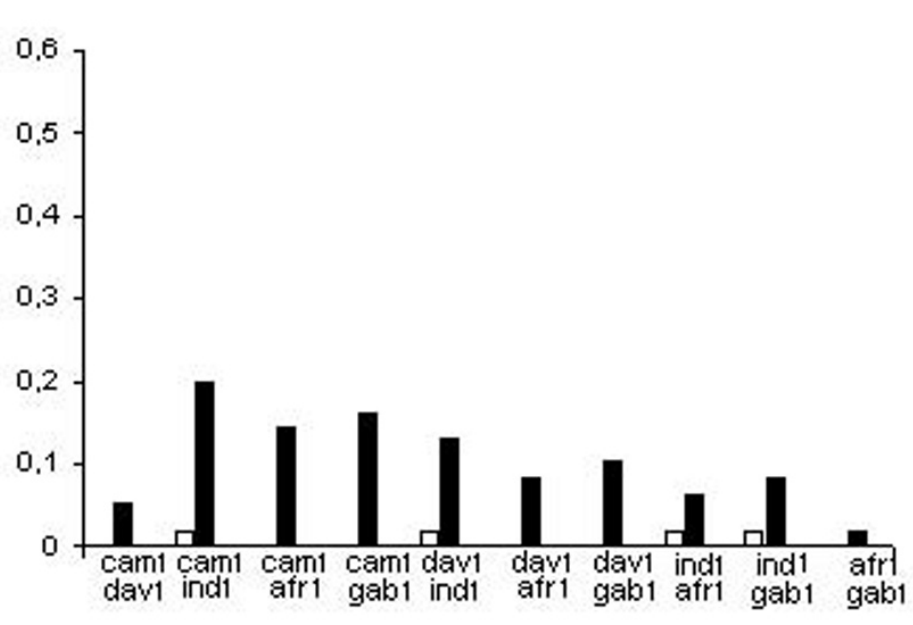

Zaprionus

clade 1

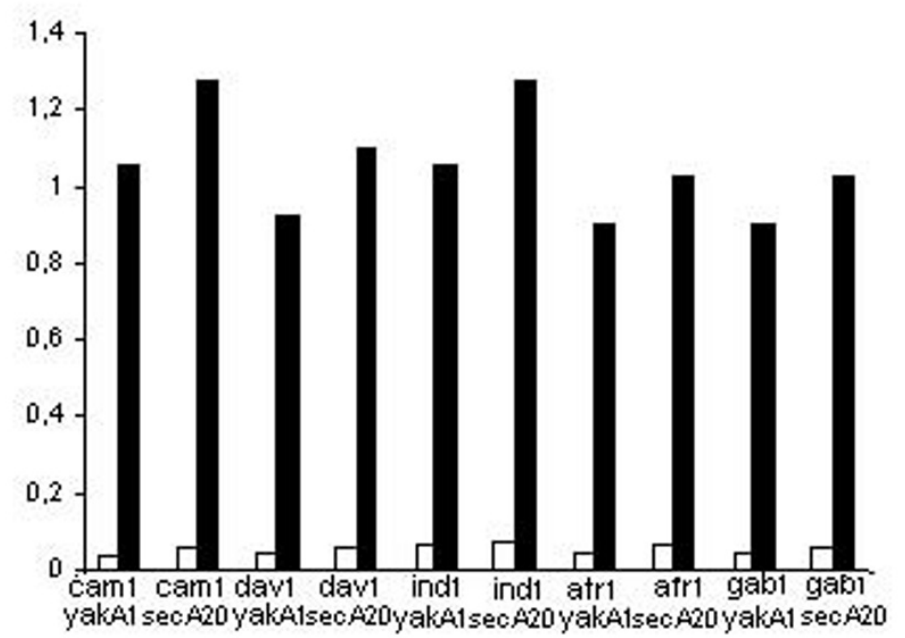

Zaprionus vs melanogaster clade 1

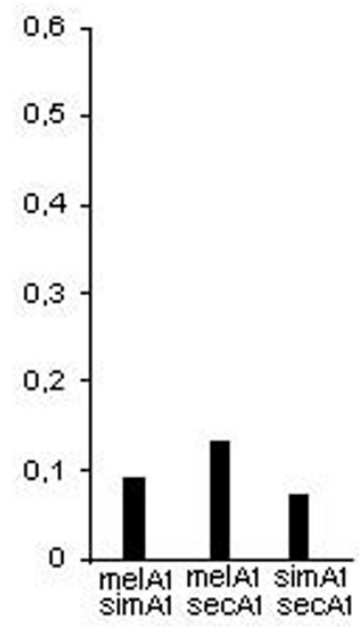

melanogaster

clade 2

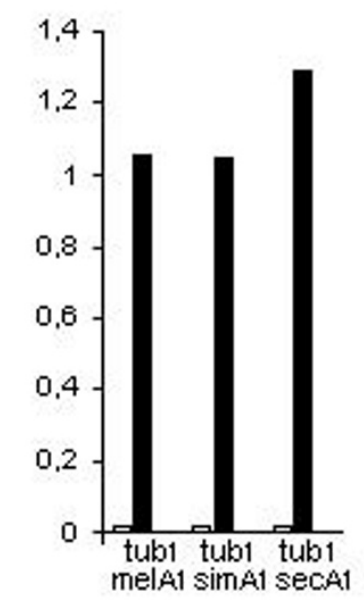

Zaprionus vs melanogaster clade 2

\section{Figure 5}

Comparative analysis of dS values of Micropia and Gpdh. Comparative analyses of the dS values between Micropia and Gpdh of Zaprionus and melanogaster species. Comparison with only black column indicates that the synonymous sites of Micropia are invariable. tub: Z. tuberculatus, cam: Z. camerounensis, dav: Z. davidi, gab: Z. gabonicus, afr: $Z$. africanus, ind: $Z$. indianus, mel: D. melanogaster, sim: D. simulans, sec: D. sechellia, yak: D. yakuba.

the Gpdh. For instance, the dS between Z. tuberculatus and the melanogaster subgroup sequences in clade 1 were from 61 (vs D. simulans) to 76 (vs D. sechellia) times lower than that those for the Gpdh. Those for the D. yakubaA1 sequence were from 18 (vs $Z$. indianus 1 ) to 31 (vs $Z$. camerounensis 1 ) times lower. In addition, dS was zero in some comparisons, e.g. those among the $Z$. camerounensis,
$Z$. davidi, Z. africanus and Z. gabonicus sequences of clade 1 . The high conservation in the synonymous sites of the RNAseH sequences favors the hypothesis that their low divergence is due to recent acquisitions of Micropia in the Zaprionus and melanogaster genomes, and that HT has shaped its evolution, as already proposed for the Gypsy retroelement. 


\section{Network trees corroborate the inference of HT}

Network reconstructions were used as an additional approach to test the hypothesis that HT has shaped the evolution of Gypsy and Micropia in Zaprionus and melanogaster species. Since TE sequences can be considered as populations of sequences that share a common ancestor, this type of phylogeny lets one propose possible donors and receptors species, and to reconstruct the TE dispersion routes by the proposition of the ancestral nodes.

The Gypsy network indicated three clusters of sequences (Figure 6a), which correspond to the S1 subfamily and the two variants of the S2 subfamily (S2V1 and S2V2). Moreover, the S1 subfamily was closer to S2V2 than to S2V1, which indicates that S2V1 diversified from S2V2. The presence of median vectors - that correspond to a hypothetical (in theory ancestral) or unsampled sequences connecting the Zaprionus and melanogaster sequences suggests that the HT events occurred in the ancestors of the sequences sampled, indicating that the lateral transmissions were not recent. The network tree allowed us to see a possible HT not clearly showed by the traditional phylogeny, but evident from the dS comparisons. The $Z$. davidi2 sequence is the ancestral node of the $Z$. africanus 1 sequence (Figure 6b), suggesting a single HT event from $Z$. davidi to $Z$. africanus. The network reconstruction also let the D. yakubaA6 sequence be included as a S2V2 member.

Network reconstruction for Micropia clustered the sequences of D. yakuba, D. melanogaster, Z. camerounensis, $Z$. davidi, $Z$. indianus and $Z$. africanus with central median vectors, suggesting that high sequence conservation was not due to HTs involving the species analyzed, but probably one or more donor species not sampled (Figure 6c). The D. melanogasterA3 sequence connects the median vectors with a large cluster that harbors D. sechellia, D. simulans and D. melanogaster sequences, which in turn links a median branch to the $Z$. tuberculatus sequences. This arrangement indicates at least two more recent transfers, one from D. melanogaster to D. simulans and/or D. sechellia or even to the ancestor of those sister species (corroborated by the low dS values), and a second from any of these species to $Z$. tuberculatus. In addition, the branch of the $D$. sechelliaA20 sequence with a median vector that links the Z. camerounensis, $Z$. davidi, Z. indianus and Z. africanus sequences suggest an additional HT involving the ancestor of $D$. sechellia. However, the absence of geographic sharing between $D$. sechellia and Zaprionus species means that this proposal should be viewed cautiously. An alternative explanation could be a high rate of evolution of the D. sechelliaA20 sequence, followed by random convergent evolution.

\section{$H T$ inference and estimation of the divergence time between TE sequences}

To reinforce the HT hypothesis, the divergence times of both retroelements were calculated using the molecular clock (see Material and Methods). The divergence estimates for Gypsy sequences showed that the HTs may have started around 11.2 MYA (Table 4). Regarding the S1 subfamily, two HTs can be proposed. The first may have occurred from a $D$. melanogaster $/ D$. simulans ancestor to $Z$. tuberculatus about 3.8-8.8 MYA, prior to the time of $D$. melanogaster/D. simulans splitting (2-3 MYA [2]). This event was followed by a transfer between $D$. melanogaster and D. erecta (up to 3.1 MYA), species that share a common ancestor at 8-15 MYA [2]. Although an alternative hypothesis of HT between $D$. simulans and D. erecta can be formulated on the basis of the phylogeny branching, the greater difference in dS comparisons between the pair $D$. erecta/D. melanogaster than the pair $D$. erecta/D. simulans (Figure 3) reinforces the first hypothesis.

The Gypsy S2V1 sequences seem to have been involved at least in four HT events. We suggest that D. erecta was the donor of S2V1 in a more ancient transfer to $Z$. tuberculatus (occurred about 5.2-11.2 MYA), followed by introduction into the $Z$. camerounensis/Z. davidi ancestor (1.2-6 MYA), and lately into D. yakuba (2.5-3.8 MYA) and D. melanogaster (2.5-3.9 MYA). The transfer to Z. camerounensis/Z. davidi probably occurred in their ancestor, because these species diverged around 2.2 MYA [1], but the hypothesis of two independent HTs cannot be disregarded. The donor status of D. erecta was based on their basal positioning in the network, the repeated clustering to all the other species of this variant in the phylogeny, the divergence times between $D$. erecta and D. melanogaster/D. yakuba (8-15 MYA [2]).

The S2V2 variant is probably involved in one HT event that happened around 0.6-10.3 MYA. It could have occurred between the ancestor of the modern sequences of D. melanogaster/D. simulans/D. sechellia and the indianus/davidi complexes. Alternatively, the HT could have happened in each complex separately, since the davidi complex diverged about 0.8 MYA [1]. The HT between the D. melanogaster/D. simulans/D. sechellia ancestor and the indianus complex ancestor is a broader scenario than the HT proposed between $Z$. indianus and D. simulans [18], exemplifying the importance of analyzing as many species as possible before inferring horizontal transfers [22]. Finally, the time of divergence also corroborates the HT from $Z$. davidi to $Z$. africanus S2V2 sequences, as seen in the network tree, which probably occurred 1.9 MYA, subsequent to the divergence of these species (2.6 to 4.1 MYA [1]). 


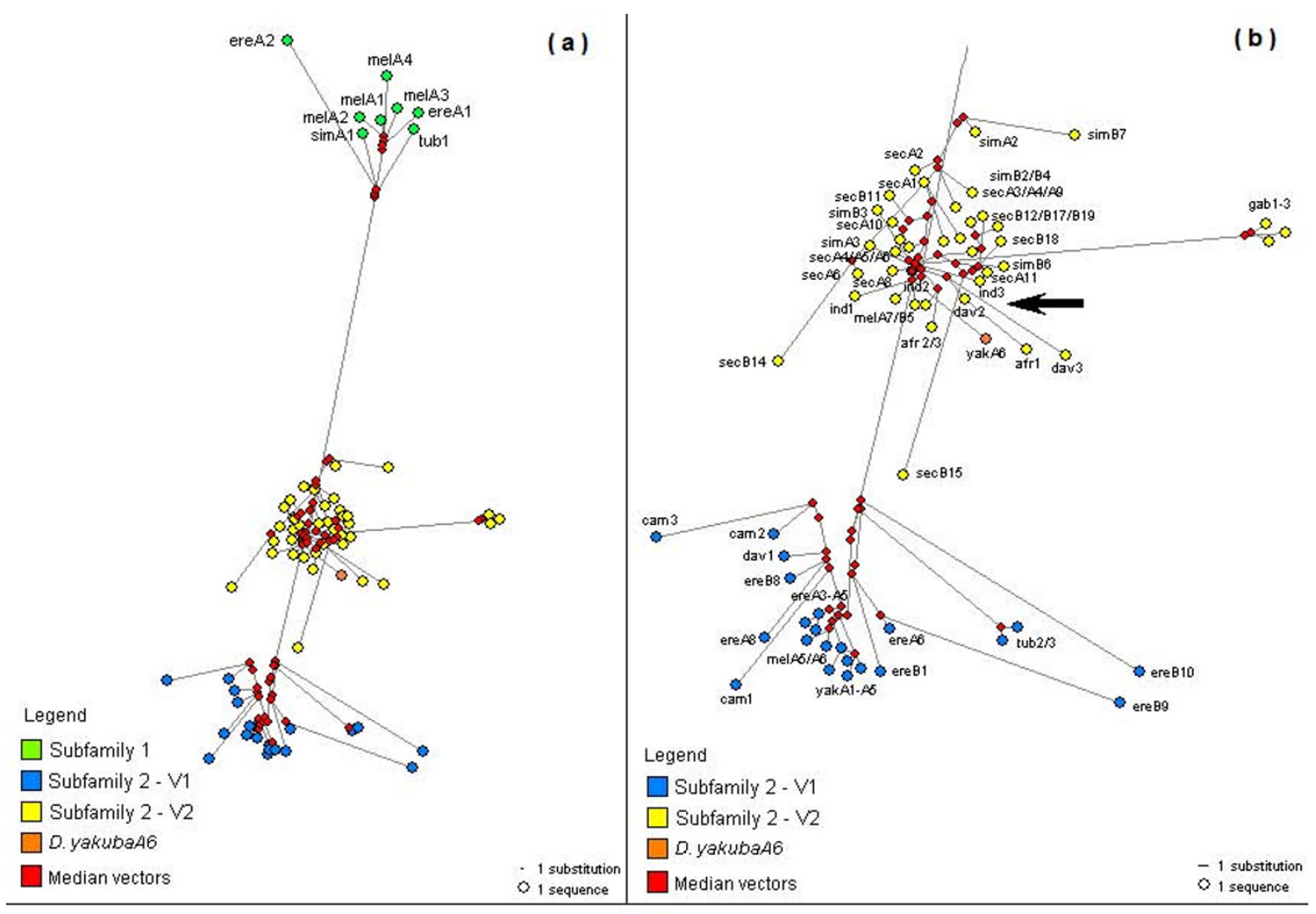

(c)

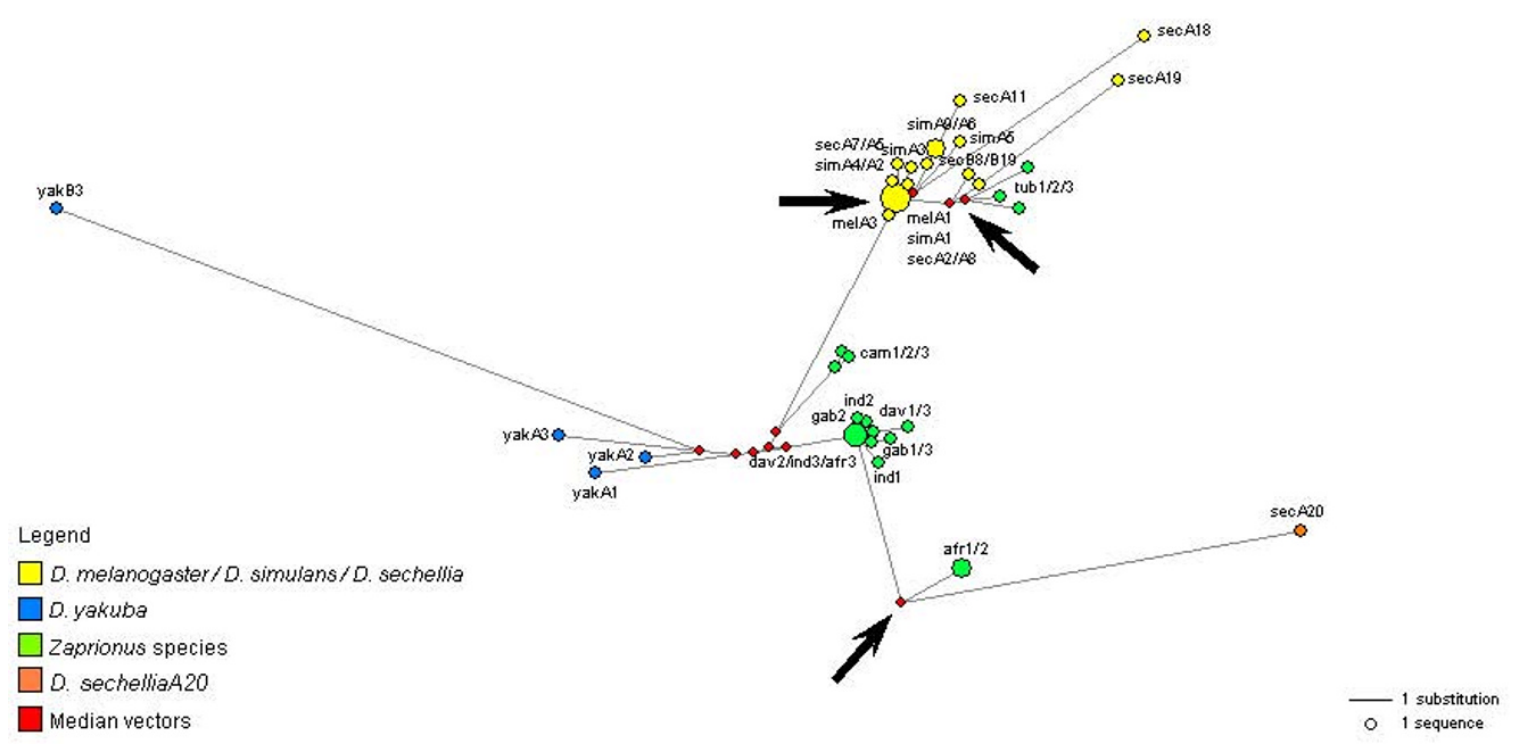

Figure 6

Network reconstructions. Median-joining network analyses for the Zaprionus and the melanogaster species. The size of each circle denotes the number of sequences grouped together. (a) Network for the Gypsy retroelement. Each group obtained in the phylogenetic tree is differently colored. (b) Detail of the Gypsy Subfamily 2. (c) Network for the Micropia retroelement. The sequences are colored according the clustering in the phylogenetic tree. The black arrows show the HT events evidenced by network trees. 
Table 4: Time of divergence and horizontal transfer events identified for the Gypsy and Micropia retroelements

\begin{tabular}{|c|c|c|}
\hline Species I & Species 2 & MYA \\
\hline \multicolumn{3}{|l|}{ Gypsy } \\
\hline \multicolumn{3}{|l|}{ Subfamily I } \\
\hline D. melanogaster/D. simulans* & Z. tuberculatus I & $3.8-8.8$ \\
\hline D. melanogaster & D. erecta & $0-3.1$ \\
\hline \multicolumn{3}{|l|}{ Subfamily 2 - VI } \\
\hline D. erecta & Z. tuberculatus & $5.2-11.2$ \\
\hline D. erecta & Z. davidi/Z. camerounensis* & $1.2-6.0$ \\
\hline D. erecta & D. yakuba & $2.5-3.8$ \\
\hline D. erecta & D. melanogaster & $2.5-3.9$ \\
\hline \multicolumn{3}{|l|}{ Subfamily 2 - V2 } \\
\hline D. melanogaster/D. simulans/D. sechellia* & indianus complex/Z. davidi* & $0.6-10.3$ \\
\hline Z. davidi & Z. africanus & $1.9 \#$ \\
\hline \multicolumn{3}{|l|}{ Micropia } \\
\hline ? & D. melanogaster & 0 \\
\hline$?$ & D. yakuba & $2.3-3.2$ \\
\hline ? & indianus complex & $0-1.6$ \\
\hline$?$ & Z. davidi & 0 \\
\hline$?$ & Z. camerounensis & 0 \\
\hline D. melanogaster & D. simulans/D. sechellia & $0 \#$ \\
\hline D. melanogaster or D. simulans/D. sechellia* & Z. tuberculatus & $0.8-1.5^{\#}$ \\
\hline
\end{tabular}

Symbols: *: Species ancestor. \#: Time of divergence calculated using dS pairwise comparisons between sequences connected by the network phylogeny.

Comparing the divergence times between the Micropia retroelement of the Zaprionus and melanogaster sequences with those of Gypsy, the HTs of Micropia might have started more recently than those of Gypsy (Micropia HTs: 3.2 MYA, Gypsy HTs: 11.2 MYA). The invariance of the synonymous sites between several pairwise comparisons [Additional file 8] makes it difficult to date these events, but the highest dS levels indicate that, of the species we have analyzed, D. yakuba and the indianus complex species were the first to be invaded by Micropia. So, the evolutionary analyses lead to the inference that Micropia diverged after the splitting of their species hosts, suggesting that at least an unknown species might have transferred Micropia sequences independently in five HTs to D. melanogaster, D. yakuba (2.3-3.2 MYA), Z. camerounensis, Z. davidi and the indianus complex (up to 1.6 MYA). Species of neither the repleta nor the cardini species group are good candidates, since they harbor Micropia retrotransposons of a different family $[29,30]$. Hence, the probable donor(s) might have been a non-studied species. Based mainly on the network tree, two other HT events can be proposed. $D$. melanogaster may have been the donor of Micropia to the D. simulans and D. sechellia, or even to their ancestor. However, introgression cannot be excluded in this case. In turn, one of these three species might have been involved in the transmission of Micropia to Z. tuberculatus, about 0.8-1.5 MYA.

\section{Discussion}

This survey of the Gypsy and Micropia in seven species of Zaprionus indicates that they are widely distributed and transcriptionally active only in species of subgenus Zaprionus. Additionally, the evolutionary analyses demonstrate that these two retroelements are closely related to those of the melanogaster species subgroup, and that their histories might have been repeatedly marked by events of interand intra-subgenus HTs. Several pieces of evidence have shown that the phenomenon of HT is frequent in eukaryotes [22,37-39]. It is important to note that the HTs proposed here were assumed only when the species involved in the event shared geographic, temporal and ecological environments, and when three separate pieces of evidence suggested their occurrence: (i) lower dS values between the TE sequences than the Gpdh sequences, (ii) incongruence between host and TE phylogenies, and (iii) last common ancestor of TE more recent than that of the species.

The studies of Micropia and Gypsy in subgenus Zaprionus species highlight the HT events as an alternative evolutionary mechanism of the retroelements evolution. Among the HTs inferred for Gypsy, four events seemed to involve the Zaprionus and melanogaster species, three involved only the melanogaster group species, and only one the Zaprionus species. For the Micropia retrotransposon, seven HTs were suggested: five between unknown donor(s) and the Zaprionus or the melanogaster species, one among the melanogaster species, and one between the Zaprionus and the melanogaster species. The 15 HTs we have proposed probably occurred during two different waves of invasions. First, the Gypsy retroelement of the melanogaster subgroup had been introduced in the Zaprionus subgenus 11 MYA. After that ( 3 MYA), a second HT 
wave involving the Micropia retroelement may have introduced it into both the Zaprionus subgenus and melanogaster subgroup from an unknown donor. After the introduction, the TEs could have been prone to transposition and re-introduction in other related species. For example, $Z$. davidi has donated their Gypsy sequence to $Z$. africanus after had received it from an ancestor of D. melanogaster, D. simulans and D. sechellia.

According to the TE evolutionary cycle, the TE history could start from HT events, followed by the initial transpositional burst in the new host, and then the accumulation of defective copies along with host-directed epigenetic silencing [40]. The last step could lead to the loss of mobility and, finally, to molecular erosion by random mutations. The high frequency of HTs, combined with the transcriptional activity and low insertion numbers of Gypsy and Micropia retroelements in the Zaprionus species subgenus indicates that these elements remain at the first or second steps of the evolutionary life history of the TEs, i.e. the invasion or the genomic spread period. A question that arises is whether these elements have been transferred in similar ways. HTs of Gypsy are easier to explain, since the active Gypsy env can enable its dispersion and expansion of the genomic territory, but this mechanism does not to apply to Micropia, which lacks env. Nevertheless, retroelements could have been transferred concomitantly with retrovirus infections, even Gypsy; or parasitic infestations, as for example by intracellular Wolbachia bacteria or mites [22]. Moreover, our data allowed us to propose there were HT of Gypsy and Micropia among sister species such as D. melanogaster, D. simulans and D. sechellia, although introgression could not be ruled out.

Another concern is the evolutionary process of fixation of the TEs after the horizontal introduction. These elements could share similar mechanisms of regulation, which would permit them escaping from selective forces and allow fixation after invasion. Control of Drosophila retroelement mobilization depends on the transcription rate, which is directly related to the presence of specific regulatory proteins [41-45] and the rate of RNA degradation, mediated by RNAi systems [46]. However, genetic information about the species of the genus Zaprionus is scarce. Further, studies of the Zaprionus genes involved in controlling mobilization could lead to hypotheses about the mechanisms that allow highly related sequences to be shared in the Zaprionus and melanogaster species genomes.

\section{Conclusion}

Our survey suggests that Zaprionus species have experienced waves of retroelement invasions, particularly during the last 7 million years. Species of the melanogaster subgroup might have donated their Gypsy sequences to species of the Zaprionus subgenus, and these two species groups could have received the Micropia retroelement from one or more unknown donor species. After the initial introduction, these species could share their elements. Since the Zaprionus genus and the melanogaster subgroup seem to share the same age of origin and diversification in tropical Africa, as well as ecological features, our data suggest that they passed through a permissive period of transposable element invasion during the diversification period.

\section{Methods}

\section{Fly stocks and DNA extraction}

All the strains of the Zaprionus species that were used are listed in Table 1, being derived from a single female selected randomly from a mass culture kindly provided by Drs Jean David and Amir Yassin from LEGS, CNRS, France. The D. melanogaster Canton-S strain was used as positive control for the molecular analyses. Genomic DNA was extracted from 10 individuals of each strain using the phenol-chloroform method [47].

\section{PCR reactions, cloning and sequencing}

Primers 2813 (5' TTA ACT CCT AGA GTT CAT CGC TGG $\left.3^{\prime}\right)$ and 2814 (5' CAT GTA CCT GGT TAA CTA CTG ACC $\left.3^{\prime}\right)$ were used to amplify the equivalent 386 bp fragment of the D. hydei Micropia retroelement located in a highly conserved region of the RNAseH domain, between nucleotides 2813 and 3198 [31]. The Gypsy retroelement fragment was obtained using primers GYP3S2F (5' AAA GGC GAY TTG GTT GAC ACT CC 3') and GYP3S2R (5' CAR GTG GCT RGG TTG RGT GTG 3') and corresponds to a 485 bp sequence located in the 6491-6511 region of env (ORF 3) in the D. melanogaster Gypsy element [18]. Both PCR reactions were performed in a final volume of $25 \mu \mathrm{l}$, using $200 \mathrm{ng}$ of genomic DNA, $0.4 \mu \mathrm{M}$ of each primer, $160 \mu \mathrm{M}$ of each dNTP, $2 \mathrm{mM} \mathrm{MgCl}_{2}$ and $1 \mathrm{U}$ Taq Platinum polymerase (Invitrogen) in $1 \times$ PCR buffer. The Gypsy cycling parameters used for amplification have been described previously [18]. The Micropia PCR cycling parameters were: $94^{\circ} \mathrm{C}$ for $3 \mathrm{~min}$ for initial denaturation, 40 cycles of $94^{\circ} \mathrm{C}$ for $30 \mathrm{~s}, 58^{\circ} \mathrm{C}$ for $1 \mathrm{~min}$, and $72^{\circ} \mathrm{C}$ for $1 \mathrm{~min}$, followed by a final extension step at $72^{\circ} \mathrm{C}$ for 10 min. The fragments obtained were purified directly from the PCR product, using the GFX PCR DNA and Gel Band Purification Kit (GE Healthcare), and cloned with the TOPO TA Cloning Kit (Invitrogen). Three randomly chosen clones were automatically sequenced in an ABI PRISM 3100 Genetic Analyzer (Applied Biosystems/Hitashi) using the primer pair T7 and M13R.

\section{RNA extraction and RT-PCR reactions}

Heads and gonads from 10 individuals of each sex were dissected in Testis Buffer (183 mM KCl, $47 \mathrm{mM} \mathrm{NaCl}$ and $10 \mathrm{mM}$ Tris- $\mathrm{HCl} \mathrm{pH}$ 6.8). Total RNA was isolated from the dissected tissues using the TRIZOL reagent (Invitrogen) 
method and genomic DNA was eliminated from the samples by RQ1 RNase-Free DNase (Promega) according to the manufacturer's instructions. The cDNA pool was generated from the total RNAs using random primers and a High Capacity cDNA Archive Kit (Applied Biosystems) under low stringency conditions $\left(37^{\circ} \mathrm{C}\right)$. The PCR reaction conditions and cycling parameters were the same as those used for genomic DNA amplification of both retroelements. To test genomic DNA contamination in the total RNA and cDNA quality Gpdh constitutive gene amplifications were performed using the total RNA extract treated with DNAse and the cDNA pool as templates, respectively. The control PCR reactions were carried out using $200 \mathrm{ng}$ of total cDNA, $0.1 \mathrm{mM}$ of each dNTP, $0.4 \mu \mathrm{M}$ of the primers ZapGPDHF (5' GTT CGG CAA TTG AAC CAA TG 3') and ZapGPDHR (5' AGA GAG TCC GTG TGC ATG TG 3'), $2 \mathrm{mM} \mathrm{MgCl}_{2}$ and $1 \mathrm{U}$ Taq Platinum polymerase (Invitrogen) in $1 \times$ PCR buffer. The cycling parameters were: $94^{\circ} \mathrm{C}$ for $2 \mathrm{~min}$ for initial denaturation, 35 cycles of $94^{\circ} \mathrm{C}$ for $1 \mathrm{~min}, 60^{\circ} \mathrm{C}$ for $1 \mathrm{~min}$ and $72^{\circ} \mathrm{C}$ for $1 \mathrm{~min}$, and an additional extension step at $72^{\circ} \mathrm{C}$ for $10 \mathrm{~min}$. The $\mathrm{Gpdh}$ primers were designed based on the $Z$. tuberculatus Gpdh (Glycerol 3 phosphate dehydrogenase) gene ORF1 (L37039) and amplify a 337 bp sequence in this sequence.

\section{Phylogenetic analyses}

Zaprionus subgenus sequences were manipulated into BioEdit [48] and aligned with Clustal W 1.81 [49]. The most divergent clones ( $>25 \%$ ) were selected as queries for a search in the 12 Drosophila genomes using the flybase BLASTn tool http://flybase.bio.indiana.edu/blast/ and the genome database released on October $17^{\text {th }}, 2008$. The queries selected were $Z$. tuberculatus $1, Z$. tuberculatus 2 and $Z$. gabonicus 1 for Gypsy, and Z. tuberculatus 2 and $Z$. africanus 1 for Micropia. The structure of the genomic sequences were predicted using the BLAST 2 sequences and ORF Finder programs. In order to obtain only highly related and non-disrupted sequences the searches were performed with stringent parameters (e-values $>e^{-50}$ and $80 \%$ coverage). Redundant genomic sequences $(100 \%$ identical) were not included in the phylogenetic analyses in order to minimize polytomies.

Multiple alignments of the Zaprionus and the genomic sequences were used to infer the phylogenetic relationships, using the maximum likelihood (ML), neighborjoining (NJ) and maximum parsimony (MP) methods, as implemented in PhyML 3.0, MEGA 4.1 and PAUP v.4.0b10 [50-52], respectively. Branch support was calculated by bootstrap analysis consisting of 1000 replicates [53]. In the NJ and ML analyses, maximum composite likelihood (MCL) and HKY85 distances were used to estimate the divergence matrices and reconstructed trees, respectively [54,55]. A heuristic search algorithm was used for MP reconstruction. The sequences obtained were registered in the GenBank database (Table 1). Gypsy sequences of S. latifaciaeformis (AF548144, AF548153 and AF548152) and D. willistoni (AF548159, AF548176 and AF548143), and Micropia sequences of D. buzzatii (AY522351), D. hydei (AY519123), D. paranaensis (AY519124), D. mercatorum (AY519125), D. seriema (AY522346), D. gouveai (AY522353), D. antonietae (AY522345), D. serido (AY522344), D. spenceri

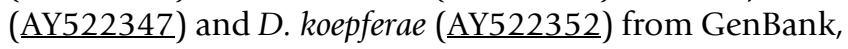
were also used in the phylogenetic reconstruction.

\section{Selection tests for Gypsy, Micropia and host gene}

The number of synonymous substitutions per synonymous site (dS), non-synonymous substitutions per nonsynonymous site $(\mathrm{dN})$, the Codon Based Z-test and $\mathrm{dN}$ :dS ratios were estimated for the Zaprionus and melanogaster sequences using the Nei-Gojobori distance with the Jukes and Cantor correction, as implemented in MEGA 4.1 [51]. The Gypsy sequences D. sechelliaA6/B14/B15, D. yakubaA6 and $D$. erectaA2/A6/B9, and the Micropia sequences $D$. simulansA3/A6, D. sechelliaA9/A18/A20 and D. yakubaA3, were excluded from those alignments because of the presence of indels ( $>2 \mathrm{bp}$ ) disrupting the open reading frames. Point ( $1 \mathrm{bp}$ ) alignment gaps were deleted prior to estimation of $\mathrm{dN}$ and $\mathrm{dS}$. The codon bias index (CBI, [56]) was estimated for each sequence using the DnaSP 4.50 program [57], where zero indicates no bias and 1 maximum bias. Sequences of exon 4 of Gpdh in Zaprionus and melanogaster subgroup species (FJ705445 to FJ705450, L37039, NM 057218, XM 002078253, XM 002089126, $\underline{\mathrm{XM} 001968825}$ and $D$. sechellia genomic sequence: scaffold_5/4016995-4017372) were used to compare the dS values of the Gypsy and Micropia sequences.

\section{Estimations of divergence time for Gypsy and Micropia}

The time of divergence of Gypsy and Micropia retrotransposons was estimated according to the molecular clock equation $r=k / 2 T$, where $r$ is the evolutionary rate $(0.011$ per site per MY, according to synonymous sites rate for genes with low codon bias in Drosophila [11]), $k$ is the dS pairwise divergence, and $\mathrm{T}$ is the divergence time between species.

\section{Median-joining networks}

The median-joining network trees were obtained using all sequences studied in the conventional phylogenies. The reconstructions were performed using DNA Aligment 1.3.0.1 and NETWORK 4.5.1.0 software [58], available at the Fluxus Technology Ltd. website. 'MP calculation' was applied for post-processing the networks, the characters were equally weighted, and the other median-joining parameters followed the software default. 


\section{List of abbreviations}

HT: horizontal transfer; LTR: long terminal repeat; TE: transposable element; env: envelope; MCL: maximum composite likelihood distance; S1: Gypsy subfamily 1; S2V1: Gypsy subfamily 2 variant 1; S2V2: Gypsy subfamily 2 variant 2; CBI: codon bias index; Gpdh: glycerol 3 phosphate dehydrogenase; dS: number of synonymous substitutions per synonymous site; $\mathrm{dN}$ : non-synonymous substitutions per non-synonymous site; ML: maximum likelihood; NJ: neighbor-joining; MP: maximum parsimony.

\section{Authors' contributions}

NS designed the study and wrote the manuscript. MAVS and PC provided laboratory facilities for experimental analyses and participated in data interpretation. CMAC conceived and supervised the project, participated in data interpretation and wrote the manuscript. All authors read and approved the final manuscript.

\section{Additional material}

\section{Additional file 1}

Transcriptional activity of Gypsy and Micropia retroelements. RTPCR was used to verify the transcriptional activity of the Gypsy env and the Micropia RNAseH genes in ovaries $(O)$, testes $(T)$ and heads $(H)$ of Zaprionus species. -: negative control with ultrapure water; +: positive control with D. melanogaster genomic DNA. Gpdh amplification was used for total RNA quality control.

Click here for file

[http://www.biomedcentral.com/content/supplementary/14712148-9-279-S1.TIFF]

\section{Additional file 2}

Insertion numbers of Gypsy and Micropia retrotransposons in Zaprionus species. The insertion numbers were indicated in the bottom of the figure. Southern blotting analyses were performed using $5 \mathrm{~g}$ of genomic DNA double digested with restriction endonucleases BamHI and MluI and probes derivate from the Gypsy env and Micropia RNAseH fragments of $\mathrm{Z}$. indianus, random primers [-P32]dCTP labelled. The nylon membranes were hybridized at $65^{\circ} \mathrm{C}$ and washed twice with $2 \times$ SSC and $0.1 \%$ SDS, and once with $0.2 \times$ SSC and $0.1 \%$ SDS for 20 min at the hybridization temperature. mel: D. melanogaster, mul: Z. multistriatus, tub: Z. tuberculatus, cam: Z. camerounensis, dav: Z. davidi, gab: Z. gabonicus, afr: Z. africanus, ind: Z. indianus.

Click here for file

[http://www.biomedcentral.com/content/supplementary/14712148-9-279-S2.TIFF]

\section{Additional file 3}

Description of the genomic sequences from the 12 Drosophila genome searches. Chromosome and nucleotide location of the Gypsy and Micropia insertions from the Drosophila genomes.

Click here for file

[http://www.biomedcentral.com/content/supplementary/14712148-9-279-S3.DOC]

\section{Additional file 4}

Structure of the Gypsy retroelement in the melanogaster subgroup genomes. Gypsy insertions in D. melanogaster ( $\mathrm{mel})$, D. simulans (sim), D. sechellia (sec), D. yakuba (yak) and D. erecta (ere). The sequences represented have at least $80 \%$ identity with the canonical element of D. melanogaster (AF033821), which is the first schematic representation. Black rectangles - long terminal repeats. Gray rectangles coding regions. Asterisk - genomic sequences interrupted by the scaffold ends or Ns. Striped rectangles - region used in the phylogenetic analyses. Click here for file

[http://www.biomedcentral.com/content/supplementary/14712148-9-279-S4.TIFF]

\section{Additional file 5}

Pairwise genetic distance among Gypsy sequences of Zaprionus, melanogaster, D. willistoni and S. latisfasciaeformis. Distances calculated by the MCL method as implemented by MEGA 4.1. The sequences were clustered according the phylogenetic clades.

Click here for file

[http://www.biomedcentral.com/content/supplementary/14712148-9-279-S5.DOC]

\section{Additional file 6}

$d N$ (below) and dS (above) values of pairwise comparisons among Zaprionus and melanogaster Gypsy sequences. Distances calculated by Nei-Gojobori method (Jukes-Cantor's correction), as implemented by MEGA 4.1.

Click here for file

[http://www.biomedcentral.com/content/supplementary/14712148-9-279-S6.DOC]

\section{Additional file 7}

$Z$ values (above) and significance P-values (below) for the CodonBased Z-test of neutrality between Gypsy sequences of Zaprionus and melanogaster species. Test performed using alternative hypothesis of non-neutrality ( $d N \quad d S)$ and Nei-Gojobori distance (Jukes-Cantor's correction). Gray cells correspond to significant $(p<0.05)$ pairwise comparisons.

Click here for file

[http://www.biomedcentral.com/content/supplementary/14712148-9-279-S7.DOC]

\section{Additional file 8}

Structure of the Micropia retroelement in the melanogaster subgroup genomes. Micropia insertions in D. melanogaster (mel), D. simulans (sim), D. sechellia (sec) and D. yakuba (yak). The sequences represented have at least $80 \%$ identity with the canonical element of $\mathrm{D}$. melanogaster (X14037), which is the first schematic representation. Black rectangles - long terminal repeats. Gray rectangles - coding regions. Asterisk - genomic sequences interrupted by the scaffold ends or Ns. Striped rectangles - region used in the phylogenetic analyses.

Click here for file

[http://www.biomedcentral.com/content/supplementary/14712148-9-279-S8.TIFF]

\section{Additional file 9}

Pairwise genetic distance among Micropia sequences of Zaprionus, melanogaster and repleta species. Distances calculated by the MCL method as implemented by MEGA 4.1.

Click here for file

[http://www.biomedcentral.com/content/supplementary/14712148-9-279-S9.DOC] 


\section{Additional file 10}

$d N$ (below) and dS (above) values of pairwise comparisons between Micropia sequences of Zaprionus and melanogaster species. Distances calculated by the Nei-Gojobori method with Jukes-Cantor's correction as implemented by MEGA 4.1 .

Click here for file

[http://www.biomedcentral.com/content/supplementary/14712148-9-279-S10.DOC]

\section{Additional file 11}

$Z$ values (above) and significance P-values (below) for the CodonBased Z-test of selection between Micropia sequences of Zaprionus and melanogaster species. Test performed using alternative hypothesis of non-neutrality $\left(\begin{array}{ll}d N & d S\end{array}\right)$ and Nei-Gojobori distance (Jukes-Cantor's correction). Gray cells correspond to significant $(p<0.05)$ pairwise comparisons.

Click here for file

[http://www.biomedcentral.com/content/supplementary/14712148-9-279-S11.DOC]

\section{Acknowledgements}

We gratefully acknowledge funding from the CAPES-COFECUB International Cooperation Program (to NS, CMAC and PC), FAPESP (MAVS - 04/ 0285 I-9), CNPq (CMAC and MAVS). NS was receipt of a CNPq fellowship. We thank J. David and A. Yassin for providing the Zaprionus strains, F. Lemeunier for the technical help, and the anonymous reviewers for their critical comments.

\section{References}

I. Yassin A, Araripe LO, Capy P, Da Lage JL, Klaczko LB, Maisonhaute C, Ogereau D, David JR: Grafting the molecular phylogenetic tree with morphological branches to reconstruct the evolutionary history of the genus Zaprionus (Diptera: Drosophilidae). Mol Phylogenet Evol 2008, 47:903-I5.

2. Lachaise D, Silvain JF: How two Afrotropical endemics made two cosmopolitan human commensals: the Drosophila mela nogaster - D. simulans palaeogeographic riddle. Genetica 2004, 120:17-39.

3. Pélandakis M, Solignac M: Molecular phylogeny of Drosophila based on ribosomal RNA sequences. J Mol Evol 1993, 37:525-543

4. Kwiatowski J, Skarecky D, Bailey K, Ayala FJ: Phylogeny of Drosophila and related genera inferred from the nucleotide sequence of the $\mathbf{C u}$, Zn Sod gene. I Mol Evol 1994, 38:443-454.

5. Russo CAM, Takezaki N, Nei M: Molecular phylogeny and divergence times of drosophilid species. Mol Biol Evol 1995, | 2:39|-404.

6. Remsen J, De Salle R: Character congruence of multiple data partitions and the origin of the Hawaiian Drosophilidae. Mol Phylogenet Evol 1998, 9:225-235.

7. Kwiatowski J, Ayala FJ: Phylogeny of Drosophila and related genera: conflict between molecular and anatomical analyses. Mol Phylogenet Evol 1999, 13:319-328.

8. Tatarenkov A, Kwiatowski J, Skarecky D, Barrio E, Ayala FJ: On the evolution of Dopa decarboxylase (Ddc) and Drosophila systematics. J Mol Evol 1999, 48:445-462.

9. Da Lage JL, Kergoat GJ, Maczkowiak F, Silvain JF, Cariou ML, Lachaise $\mathrm{D}$ : A phylogeny of Drosophilidae using the amyrel gene: questioning the Drosophila melanogaster species group boundaries. J Zoolog Syst Evol Res 2007, 45:47-63.

10. Dalton R: A fly by any other name. Nature 2009, 457:368.

II. Tamura K, Subramanian S, Kumar S: Temporal patterns of fruit fly (Drosophila) evolution revealed by mutation clocks. Mol Biol Evol 2004, 21 :36-44.
12. Cizeron G, Lemeunier F, Loevenbruck C, Brehm A, Biémont C: Distribution of the retrotransposable element 4 I 2 in Drosophila species. Mol Biol Evol 1998, I5: I589-1599.

13. Montchamp-Moreau C, Ronsseray M, Jacques M, Lehmann M, Anxolabéhère $D$ : Distribution and conservation of sequenceshomologous to the I73 I retrotransposon in Drosophila. Mol Biol Evol 1993, 10:79|-803.

14. Moschetti R, Caggese C, Barsanti P, Caizzi R: Intra- and interspecies variation among Bari-I elements of the melanogaster species group. Genetics 1998, I 50:239-250.

15. Maruyama K, Hartl DL: Evolution of the transposable element mariner in Drosophila species. Genetics 1991, 128:319-329.

16. Brunet F, Godin F, David JA, Capy P: The mariner transposable element in the Drosophilidae family. Heredity 1994 73:377-385.

17. Brunet F, Godin F, Bazin C, Capy P: Phylogenetic analysis of Mos I -like transposable elements in the Drosophilidae. I Mol Evol 1999, 49:760-768.

18. Herédia F, Loreto EL, Valente VL: Complex evolution of gypsy in drosophilid species. Mol Biol Evol 2004, 2 I : I83 I-I842.

19. McDonald JF, Matyunina LV, Wilson S, Jordan IK, Bowen NJ, Miller WJ: LTR retrotransposons and the evolution of eukaryotic enhancers. Genetica 1997, 100:3-13.

20. Vidal NM, Ludwig A, Loreto ELS: Evolution of Tom, 297, I7.6 and rover retrotransposons in Drosophilidae species. Mol Genet Genomics 2008, 282:353-362.

21. Almeida LM, Carareto CM: Sequence heterogeneity and phylogenetic relationships between the copia retrotransposonin Drosophila species of the repleta and melanogaster groups. Genet Sel Evol 2006, 38:535-550.

22. Loreto EL, Carareto CM, Capy P: Revisiting horizontal transfer of transposable elements in Drosophila. Heredity 2008, 100:545-554.

23. De Setta N, Carareto CMA: Screening for transposable elements in South America invasive species Zaprionus indianus and Drosophila malerkotliana. Drosoph Inf Serv 2007, 90:96-99.

24. Lloréns C, Futami R, Bezemer D, Moya A: The Gypsy Database (GyDB) of mobile genetic elements. Nucleic Acids Res 2008, 36:D38-D46.

25. Eickbush TH: Origin and evolutionary relationships of LTR retroelements. In The evolutionary biology of viruses Edited by: Morse SS. New York, NY: Raven; 1994: I II-I57.

26. Llorens JV, Clark JB, Martínez-Garay I, Soriano S, de Frutos R, Martínez-Sebastián MJ: Gypsy endogenous retrovirus maintains potential infectivity in several species of Drosophilids. $B M C$ Evol Biol 2008, 8:302.

27. Boeke JD, Eickbush TH, Sandmeyer SB, Voytas DF: Metaviridae. In Virus Taxonomy Edited by: Murphy FA. New York, NY: Springer-Verlag; 1999:123-135

28. Huijser P, Kirchhoff C, Lankenau DH, Hennig W: Retrotransposonlike sequences are expressed in $\mathbf{Y}$ chromosomal lampbrush loops of Drosophila hydei. J Mol Biol 1988, 203:689-697.

29. Almeida LM, Carareto CMA: Identification of two subfamilies of micropia transposable element in species of the repleta group of Drosophila. Genetica 2004, I 2 I: I55-164.

30. Cordeiro J, Robe LJ, Loreto EL, Valente VL: The LTR retrotransposon micropia in the cardini group of Drosophila (Diptera: Drosophilidae): a possible case of horizontal transfer. Genetica 2008, I34:335-344

31. Lankenau S, Corces GV, Lankenau DH: The Drosophila micropia retrotransposon encodes a testis-specific antisense RNA complementary to reverse transcriptase. Mol Cell Biol 1994, I 4: I764- I775.

32. Ludwig A, Valente VL, Loreto EL: Multiple invasions of Errantivirus in the genus Drosophila. Insect Mol Biol 2008, I 7: I I3-I I 2.

33. Alberola TM, De Frutos R: Molecular structure of a gypsy element of Drosophila subobscura (gypsyDs) constituting a degenerate form of insect retroviruses. Nucleic Acids Res 1996 , 24:914-923.

34. Mizrokhi LJ, Mazo AM: Cloning and analysis of the mobile element gypsy from D. virilis. Nucleic Acids Res 1991, 19:913-916.

35. Mejlumian L, Pélisson A, Bucheton A, Terzian C: Comparative and functional studies of Drosophila species invasion by the gypsy endogenous retrovirus. Genetics 2002, I 60:20 I-209. 
36. Jordan IK, McDonald JF: Interelement selection in the regulatory region of the copia retrotransposon. J Mol Evol 1998, 47:670-676.

37. Pace JK II, Gilbert C, Clark M, Feschotte C: Repeated horizontal transfer of a DNA transposon in mammals and other tetrapods. Proc Natl Acad Sci USA 2008, 105: I 7023- 17028.

38. Roulin A, Piegu B, Fortune PM, Sabor F, D'Hont A, Manicacci D, Panaud $O$ : Evidence of multiple horizontal transfers of the long terminal repeat retrotransposon RIREI within the genus Oryza. Plant J 2008, 53:950-959.

39. Cheng $X$, Zhang D, Cheng Z, Keller B, Ling HQ: A new family of Ty-copia -like retrotransposons originated in the tomato genome by a recent horizontal transfer event. Genetics 2009, I8I:1 I83-II93.

40. Pinsker W, Harina E, Hagemann S, Miller WJ: The evolutionary life history of $P$ transposons: from invaders to domesticated neogenes. Chromosoma 200I, II 0:148-158.

41. Cavarec L, Heidmann T: The Drosophila copia retrotransposon contains binding sites for transcriptional regulation by homeoproteins. Nucleic Acids Res 1993, 21:504 I-5049.

42. Bhadra U, Pal-Bhadra M, Birchler JA: Role of the male specific lethal (msl) genes in modifying the effects of sex chromosomal dosage in Drosophila. Genetics 1999, 152:249-268.

43. Pal-Bhadra M, Bhadra U, Birchler JA: Role of multiple trans-acting regulators in modifying the effect of the retrotransposon copia on host gene expression in Drosophila. Mol Gen Genet 1998, 259:198-206.

44. Cavarec $L$, Jensen $S$, Heidmann $T$ : Identification of a strong transcriptional activator for the copia retrotransposon responsible for its differential expression in Drosophila hydei and melanogaster cell lines. Biochem Biophys Res Commun 1994 203:392-399.

45. Tulin A, Stewart D, Spradling AC: The Drosophila heterochromatic gene encoding poly(ADP-ribose) polymerase (PARP) is required to modulate chromatin structure during development. Genes Dev 2002, 16:2108-2119.

46. Siomi MC, Saito K, Siomi H: How selfish retrotransposons are silenced in Drosophila germline and somatic cells. FEBS Lett 2008, 582:2473-2478.

47. Jowett T: Preparation of nucleic acids. In Drosophila: A Practical Approach Edited by: Roberts DB. Oxford: IRL Press; 1986:275-277.

48. Hall TA: BioEdit: a user-friendly biological sequence alignment editor and analysis program for Windows 95/98/NT. Nucleic Acids Symp Ser 1999, 41 : 95-98.

49. Thompson JD, Higgins DG, Gibson TJ: CLUSTAL W: improving the sensitivity of progressive multiple sequence alignment through sequence weighting, position-specific gap penalties and weight matrix choice. Nucleic Acids Res 1994, 22:4673-4680.

50. Guindon S, Gascuel O: A simple, fast, and accurate algorithm to estimate large phylogenies by maximum likelihood. Syst Biol 2003, 52:696-704.

51. Tamura K, Dudley J, Nei M, Kumar S: MEGA4: Molecular Evolutionary Genetics Analysis (MEGA) software version 4.0. Mol Biol Evol 2007, 24:1596-1599.

52. Swofford D: PAUP: phylogenetic analysis using parsimony: Version 4.0b I 0. Washington, DC: Smithsonian Institution; 1997.

53. Felsenstein J: Confidence limits on phylogenies: an approach using the bootstrap. Evolution 1985, 39:783-791.

54. Tamura K, Nei M, Kumar S: Prospects for inferring very large phylogenies by using the neighbor-joining method. Proc Natl Acad Sci USA 2004, I01: I 1030- I 1035.

55. Hasegawa M, Kishino H, Yano T: Dating of the human-ape splitting by molecular clock of mitochondrial DNA. J Mol Evol 1985, 21:160-174.

56. Morton BR: Chloroplast DNA codon use: evidence forselection at the psb locus based on tRNA availability. J Mol Evol 1993, 37:273-280.

57. Rozas J, Sánchez-DelBarrio JC, Messeguer X, Rozas R: DnaSP, DNA polymorphism analyses by the coalescent and other methods. Bioinformatics 2003, 19:2496-2497.

58. Bandelt $\mathrm{HJ}$ : Median-joining networks for inferring intraespecific phylogenies. Mol Biol Evol 1999, 16:37-48.

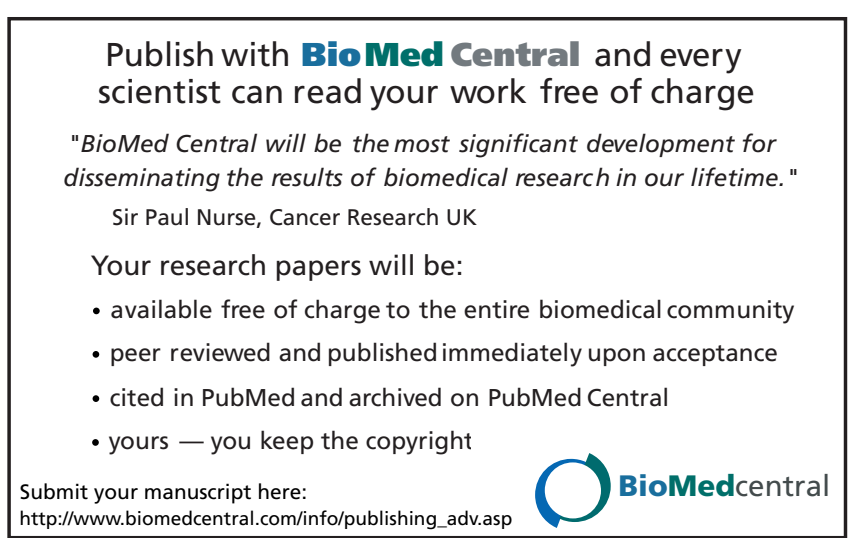

